\title{
Identification of epigenetic factors regulating the mesenchyme to epithelium transition by RNA interference screening in breast cancer cells
}

Jean-Marc Gregoire, Laurence Fleury, Clara Salazar-Cardozo, Frédéric Alby, Véronique Masson, Paola Barbara Arimondo and Frédéric Ausseil ${ }^{*}$

\begin{abstract}
Background: In breast cancer, the epithelial to mesenchyme transition (EMT) is associated to tumour dissemination, drug resistance and high relapse risks. It is partly controlled by epigenetic modifications such as histone acetylation and methylation. The identification of genes involved in these reversible modifications represents an interesting therapeutic strategy to fight metastatic disease by inducing mesenchymal cell differentiation to an epithelial phenotype.

Methods: We designed a siRNA library based on chromatin modification-related to functional domains and screened it in the mesenchymal breast cancer cell line MDA-MB-231. The mesenchyme to epithelium transition (MET) activation was studied by following human E-CADHERIN (E-CAD) induction, a specific MET marker, and cell morphology. Candidate genes were validated by studying the expression of several differential marker genes and their impact on cell migration.

Results: The screen led to the identification of 70 gene candidates among which some are described to be, directly or indirectly, involved in EMT like ZEB1, G9a, SMAD5 and SMARCD3. We also identified the DOT1L as involved in EMT regulation in MDA-MB-231. Moreover, for the first time, KAT5 gene was linked to the maintenance of the mesenchymal phenotype.
\end{abstract}

Conclusions: A multi-parametric RNAi screening approach was developed to identify new EMT regulators such as KAT5 in the triple negative breast cancer cell line MDA-MB-231.

Keywords: Epithelium, Mesenchyme, Transition, RNAi, Screening, DOT1L, KAT5/Tip60

Abbreviations: CM, Chromatin modification; DDR, DNA damage response; E-CAD, E-CADHERIN; EMT, Epithelial to mesenchyme transition; ESCs, Embryonic stem cells; HMBS, Hydroxymethylbilane synthase; IPO8, Importin 8; iPSCs, Induced pluripotent stem cells; MAD, Median absolute deviation; MET, Mesenchyme to epithelium transition; N-CAD, N-CADHERIN; OCLN, Occludin; PPIA, Peptidylprolyl isomerase A; RNAi, RNA interference; TICs, Tumour initiating cells; TNBC, Triple-negative breast cancer; TSPAN13, Tetraspanin 13

\footnotetext{
* Correspondence: frederic.ausseil@pierre-fabre.com

Unité de Service et de Recherche CNRS-Pierre Fabre n³388 ETaC, CRDPF,

3 avenue $H$. Curien, BP 13652, 31035, Toulouse cedex 01, France
} 


\section{Background}

In breast tumours, the epithelium to mesenchyme transition (EMT) is associated to early metastatic cell dissemination, drug resistance and high relapse risks [1]. During this epithelial cell dissemination, primary tumours acquire a mesenchymal phenotype [2]. Cytoskeletal rearrangements resulting in loss of cell polarity and morphology properties improve the migratory and invasive features of the cells [3]. Relapse risks are frequent for particularly aggressive cancer forms which display EMT and invasive properties often associated to $\mathrm{CD} 44^{\text {high }} / \mathrm{CD} 24^{- \text {llow }}$ phenotype and present tumour initiating cell (TICs) features like auto-renewing and chemo-resistance [4-6]. Interestingly, the analysis of clinical samples indicates that metastases often closely look like the primary tumour in morphology and gene expression profile suggesting that the redifferentiation of the metastasizing cell may occur via a mesenchymal to epithelial transition (MET) [7]. Indeed, after MET, the cells look and expand to form a secondary tumour [8-10]. Strikingly, changes in cellular characteristics during a bona fide MET are to a large extent dependent on the upregulation of E-CAD and the repression of N-CADHERIN (NCAD), both belonging to type- 1 transmembrane proteins class regulated by the MET program [3]. As cell dissemination and tumour initiation are linked to MET in breast cancer, the identification of the targets involved in this biological pathway is critical for the discovery of novel therapies.

The role of epigenetic mechanisms in EMT of breast cancer cells is emerging [11]. Epigenetic is composed of chromatin modification (CM) such as DNA methylation, histone post-modifications that dictates access to DNA, thereby playing a major role in the regulation of transcription, DNA recombination, replication, and repair [12]. Higher-order chromatin structure is also an important regulator of gene expression during mammalian development, lineage specification [13] and shapes the mutational landscape of cancer [14]. Since chromatin modifications are reversible, epigenetic marks constitute ideal targets for therapeutic action.

Here, we aimed at identifying the regulators involved in MET as future therapeutic targets in breast cancer. MDA-MB-231 cell line was used as mesenchymal breast cancer model and RNA interference (RNAi) was used to identify the chromatin modifying domains involved in MET. RNAi-mediated gene silencing is a valuable tool widely used in drug discovery $[15,16]$ notably in highthroughput screening $[17,18]$. A set of 729 chromatin modifying target genes were chosen according to the bioinformatic study of $\mathrm{Pu}$ et al. [19] and pools of four siRNA per target were designed.

Since E-CAD induction is a feature of MET, we followed the detection of E-CAD by fluorescence microscopy together with the change in cell morphology towards an epithelial phenotype. To confirm the siRNA hits, the expression of targeted genes and their impact on cell migration were measured. Thereby, the already described G9a, SMAD5 and SMARCD3 were identified to be involved in MET, as also DOT1L that has been recently published in this domain. Finally, for the first time, KAT5 was found to be involved in MET.

\section{Methods}

\section{Cell line and drug}

MDA-MB-231 cells were grown in Dulbecco's modified Eagle's medium (DMEM-GlutaMAX ${ }^{\mathrm{TM}}$-I from Gibco) supplemented with $10 \%$ fetal bovine serum (Lonza). Cells were incubated at $37{ }^{\circ} \mathrm{C}$ with $5 \% \mathrm{CO}_{2}$ and subcultured twice weekly during the experimental period.

EPZ-5676 was purchased from ChemScene (USA). A DMSO stock solution $(10 \mathrm{mM})$ was prepared and stored at $-20{ }^{\circ} \mathrm{C}$ until ready for use. Working dilutions were prepared in DMEM just before use.

\section{SiRNA and miRNAs}

The SMARTpool siRNA library (targeting 729 known and putative human chromatin modifiying genes) was purchased from Dharmacon (GE Healthcare) in ten 96well plates (80 SMARTpool siRNAs/plate). The ONTARGETplus siRNA SMARTpool against ZEB1 was purchased from Dharmacon (GE Healthcare) whereas the negative control siRNA ( $\mathrm{siScr}$ ) was purchased from Qiagen (AllStars Negative Control). The pre-miR-200a, pre-miR-200c and pre-miR Negative Control 2 were purchased from Ambion (Life Technologies) [20].

\section{siRNA screening and hits validation}

MDA-MB-231 (3,000/well) were reverse transfected in 96-well plates, in duplicate, with SMARTpool siRNA library using Lipofectamine ${ }^{\bullet}$ RNAiMAX (Invitrogen) following the manufacturer's instructions. The final concentration of each SMARTpool siRNA was $10 \mathrm{nM}$ in $100 \mu \mathrm{l}$ medium per well. After $72 \mathrm{~h}$, media were removed and cells were re-transfected (forward transfection) with SMARTpool siRNA at the same concentration as previously described. After $72 \mathrm{~h}$, media were definitively removed and cells were washed one time with PBS1x before fixation with $3.7 \%$ paraformaldehyde (Sigma-Aldrich) and permeabilization with $0.1 \%$ Triton X-100 (Sigma-Aldrich). The plates were then blocked with PBS1x containing $2 \%$ BSA plus $0.05 \%$ Tween-20 (Sigma-Aldrich) overnight at $4{ }^{\circ} \mathrm{C}$. Next, the plates were incubated with mouse anti-E-CAD antibody (1:200; BD Pharmingen) for $2 \mathrm{~h}$ at room temperature. After washing three times with PBS $1 \times$ plus $0,05 \%$ Tween 20, the plates were incubated with a mixture of Alexa Fluor 488 Donkey Anti-Mouse antibody (1:1000; Life Technologies), Texas-Red ${ }^{\circ}-\mathrm{X}$ 
Phalloidin (1:200; Life Technologies) and DAPI (1:2000; AAT Bioquest) for $1 \mathrm{~h}$ at room temperature, washed three times before analysis on the IN Cell Analyser 1000 (20x, GE Healthcare). Five fields per well were scanned and analysed. Each plate contained two positive controls (a SMART pool directed against $Z E B 1$ and a pre-miR200c) and two negative controls (cells treated with transfection reagent alone; and transfected with a scramble siRNA). For each transfection, the immunofluorescence of E-CAD was normalized to the cell number measured by DAPI staining. The data were normalized to the median signal of the plate and MAD (median absolute deviation) was used for hit selection [21]. For analysis, since the values measured for the ZEB1 positive control were between one or two MAD, hits were selected on this criteria: a MAD value superior to one. The MAD value was associated to cell morphological change analysis (Moreno-Bueno et al. [22]). For hit validation, E-CAD induction was measured by RT-qPCR and considered positive if two single siRNA out of the four of the pool were positive (Boutros et al. [23]). The significance of E-CAD induction was analysed using the Wilcoxon-Mann-Whitney test. A $p$-value $<0.05$ was considered statistically significant.

\section{RNA isolation}

After two successive transfections, cells were harvested by trypsinization and total RNA was isolated using the RNeasy plus mini kit following the manufacturer's instructions (Qiagen). The quantity and quality of the RNA were determined using the NanoDrop $2000 \mathrm{spec}-$ trophotometer (ThermoScientific).

\section{Quantitative RT-qPCR}

cDNA was synthetized from $1 \mu \mathrm{g}$ of total RNA using the SuperScript ${ }^{\circ}$ VILO $^{\text {TM }}$ cDNA Synthesis Kit according to the manufacturer's instructions (Life Technologies). QRT-PCR was performed using SYBR $^{\circ}$ Green PCR Master Mix (Applied Biosystem) and a CFX384 ${ }^{\mathrm{TM}}$ RealTime PCR Detection System (Bio-Rad). Gene expression was normalized to three endogenous control genes (hydroxymethylbilane synthase $(H M B S)$, Peptidylprolyl Isomerase A (PPIA), Importin 8 (IPO8).

PCR primers were synthetized by Eurogentec. The following primer sequences were used.

For DOT1L, 5'-GCTGCCACCAGACTGACCA-3'(forward) and 5'-TCCTAGTTACCTCCAACTGTGCC-3'(reverse); for KAT5 5'-TCCCCAGGGGGAGATAATCGAG3'(forward) and 5'-GCCAGGGGCCACTCATCTTC-3' (reverse); for E-cadherin 5'-TCCCACCACGTACAAGG GTC-3'(forward) and 5'-GGGGGCATCAGCATCAGTC A-3'(reverse); for CD24 5'-AACTAATGCCACCACCAA GG-3'(forward) and 5'-GACGTTTCTTGGCCTGAGTC3'(reverse); for TSPAN13 QuantiTect Primer Assay (Qiagen); for $H M B S$ 5'-ATACAGACGGACAGTGTGGTGG
C-3'(forward) and 5'-CCCTGTGGTGGACATAGCAATG A-3'(reverse); for PPIA 5'-GAGCACTGGAGAGAAAG GATTTGGTT-3'(forward) and 5'-CGTGTGAAGT CAC CACCCTGACA-3'(reverse); for IPO8 5'-GAGTGTGAG GGTCAAGGGGATG-3'(forward) and 5'-AAAGTGCTG CCTAATGCCAGATG-3'(reverse).

\section{Migration assays}

Migration assays were performed with the Oris ${ }^{\mathrm{TM}}$ Cell Migration Assay following the manufacturer's instructions (PLATYPUS Technologies). Briefly, after two successive transfections, cells were harvested by trypsinization and counted. For each transfection, 80.000 cells/ well were seeded and allowed to adhere for $24 \mathrm{~h}$. Stoppers were removed and the plate was incubated to permit cell migration for $24 \mathrm{~h}$. The cells were labelled with calcein AM (Life Technologies) and the fluorescence was detected using a Typhoon Trio (GE-Healthcare). The effects on cell migration were estimated by cell surface area calculation using Image J program (National Institutes of Health Image). Each experiment was done in triplicate with two independent repeats.

\section{Results and discussion}

Design of the 729 siRNA pool library

The siRNA pool library is directed against 729 known or predicted chromatin modifier genes like chromatinremodeling factors (KATs, HDACs, KMTs and KDMs), transcriptional coactivators or corepressors (Additional file 1). Substantial evidences show that the chromatin modifying factors exhibits distinct protein domains that perform specific functions, such as SET domain (a catalytic domain of many histone lysine methyl-transferases), Bromodomain (responsible for recognition of acetylated histone lysine) or Chromodomain (responsible for binding of methylated histone lysine) [24-26]. The library, which includes four independent siRNAs for each targeted gene, was designed according to an orthologybased computation analysis of the Pfam protein database looking for the protein domains involved in chromatin modification [19, 24-27]. In this study, the authors predicted 397 novels CM genes (coding for 329 proteins) in humans in addition to 398 experimentally verified ones to propose a library of genes in chromatin modification. Here, the siRNA library was generated by deleting unvalidated gene sequences and adding genes involved in DNA methylation to obtain the 729 siRNA pools library (Additional file 1 for the list of the RNAi bank).

\section{Screening strategy's steps}

To identify new chromatin modifying genes involved in the maintenance of the mesenchymal state, a four step strategy was performed (Fig. 1). The triple-negative breast cancer (TNBC) cell line model MDA-MB-231 


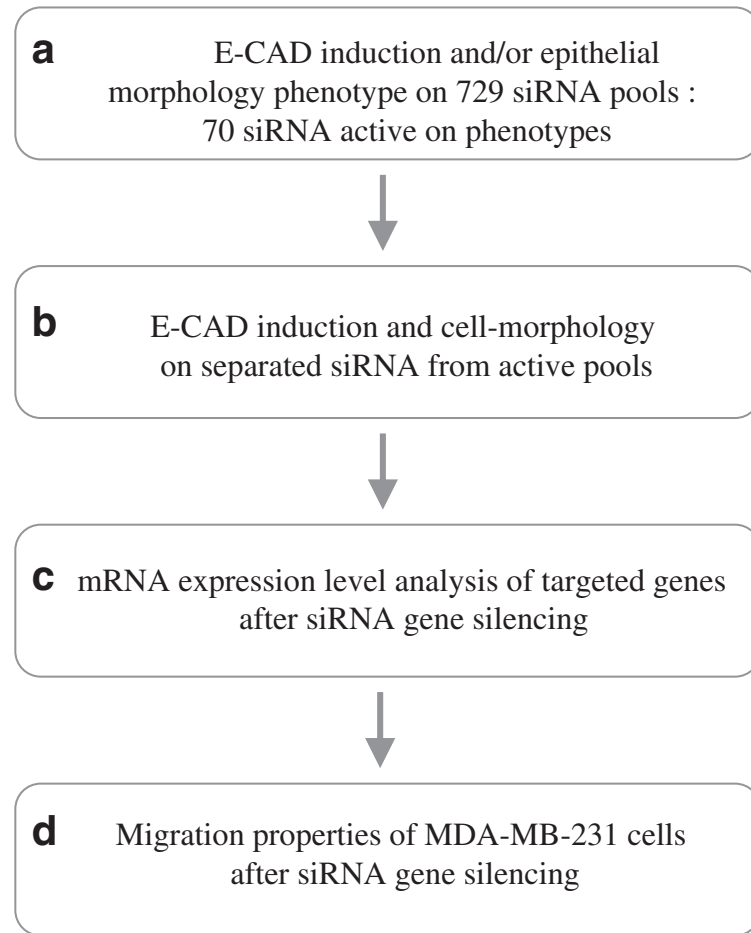

Fig. 1 Screening strategy. A four step process was used to identify MET inducer gene candidates. a The primary screening was performed on 729 siRNA pools targeting 729 genes selected for chromatin structure maintenance. From E-CAD induction measurements and cell morphology observations, 70 pools were identified. b Deconvolution analysis: E-CAD induction and cell morphology were analysed for each siRNA contained in the active pools. c Transcript quantification was done by RT-qPCR to control gene knockdown. $\mathbf{d}$ Cell migration study: the lost of the mesenchyme phenotype was associated to impaired migration capabilities for several siRNA

was chosen because it's representative of the mesenchymal-like phenotype of cancer cells and represents one of the most aggressive human cancer cells when grafted in mice $[28,29]$. Interestingly, HDACi inhibition initiates a partial MET which is associated to decreased tumorigenesis in vivo [30] indicating that by acting on the epigenetic regulation it is possible to reverse the mesenchymal phenotype. In addition, this cell line has a relatively high percentage of CD44 ${ }^{+} / \mathrm{CD} 24^{-/ \text {low }}$ cells which have been reported to have stem/progenitor cells properties [4] and enhanced invasive properties [31].

The MDA-MB-231 cells do not express, or weakly, ECAD which is silenced by methylation of its promoter [28]. The MET is partly characterized by the reactivation of E-CAD a marker of the epithelial state. Thus, the first step of the strategy consisted in screening the 729 siRNA pools on these cells to identify the pools of siRNA that induced E-CAD as followed by immunofluorescence. In parallel, epithelial cell morphology was followed by F-ACTIN immunofluorescence staining. Second, the 4
siRNA of each active pool were tested separately on both E-CAD induction and cell morphology. Third, the down-regulation of the targeted genes was confirmed by RT-qPCR. Fourth, the effect of the siRNA was further validated by inhibition of the migration properties of the cells.

\section{Cell-based assay validation}

The microRNA-200 (miR-200) family has emerged recently as important regulators of EMT/MET [32]. This family comprises five members expressed from two distinct polycistronic transcripts (miR-200b 200a 429 and miR-200c 141) and, on the basis of their 'seed' sequence [33], can be separated in two functional groups (miR-200b/200c/429 and miR-141/ 200a). The miR-200c is known to be involved in cells undergoing EMT/MET [20, 34].

The miR-200c and a miRNA negative control were used as positive and negative controls respectively. The comparison of miR-200c and miRNA negative control transfected cells in phase contrast microscopy showed a dramatic change of cell morphology, from an elongated fibroblast-like shape with pronounced cellular scattering to a cobblestone-like epithelial phenotype (Fig. 2a). RTqPCR analysis revealed a significant increase in the expression of the epithelial marker E-CAD mRNA in miR200 family (miR-200b and miR200a) transfected cells (Fig. 2b). The immunofluorescence analysis of E-CAD reinforced this result. In several cancer cell types, the miR-200 family is able to enforce an epithelial state by inhibiting the E-CAD transcriptional repressor ZEB1 $[33,35]$. In our model, cells transfected with miR-200c, or a specific SMARTpool directed against ZEB1, showed a strong E-CAD cellular membrane staining and a discrete nuclear staining whereas MDA-MB-231 cells transfected with a miRNA negative control (data not shown) or an irrelevant siRNA only showed a weak nuclear staining (Fig. 2c). As E-CAD nuclear staining was unexpected, we conducted the same experiment with a second antibody directed against E-CAD obtaining the same result (data not shown). Finally, we observed an increase in E-CAD signal and F-ACTIN staining with phalloidin clearly revealed the cuboidal phenotype, typical of epithelial cells, of miR-200c and siZEB1 transfected cells (Fig. 2c). Taken together, these experiments validate miR-200c and siZEB1 as inducers of MET in MDA-MB-231 cells.

\section{siRNA screening reveals genes potentially involved in MET}

Transfection reagent, cell number and siRNA concentrations were optimized to obtain a maximum of $20 \%$ reduction in cells viability when transfected with the irrelevant (non silencing) siRNA compared to mock- 

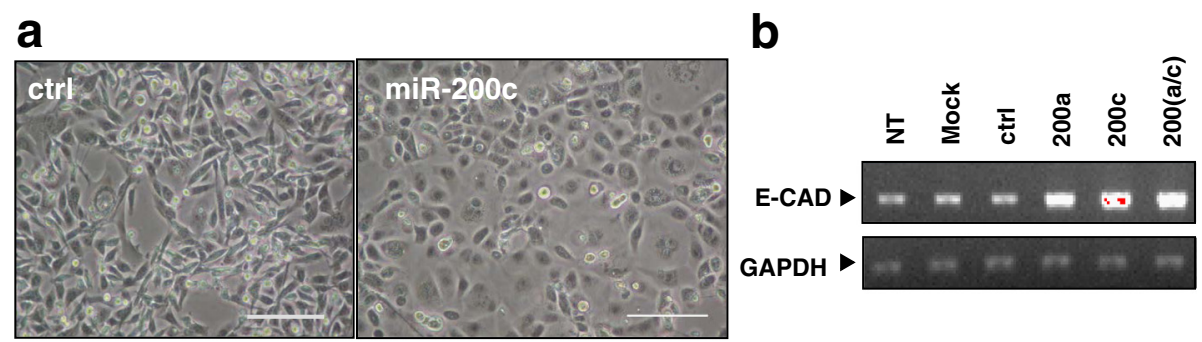

C

DAPI

E-CAD

F-actin
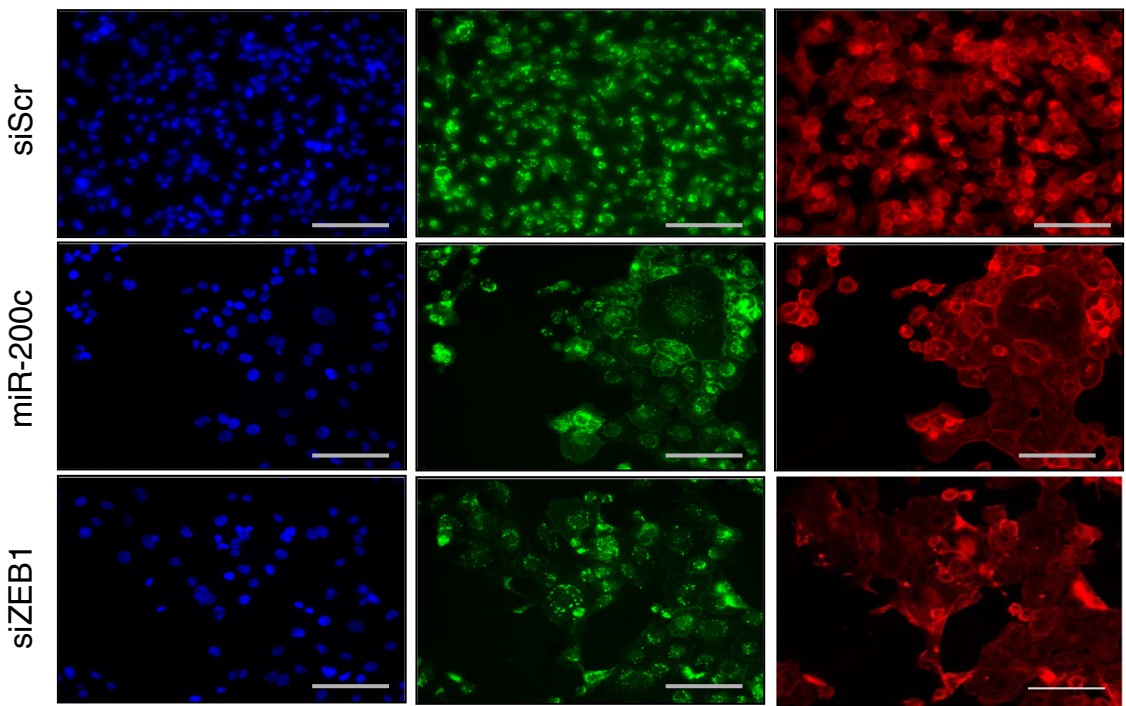

Fig. 2 Cell based assay development and validation. a MDA-MB231 cells were transfected with a pre-miRNA negative control (ctrl; 5nM) or pre-miR-200c (5nM). Phase contrast images were taken at 6 days after 2 successive transfections (magnification, $\times 10$ ). b MDA-MB-231 cells were transfected as in A with a pre-miR negative control scramble (ctrl), pre-miR-200a (200a), pre-miR-200c, mix of pre-miR200a + pre-miR-200c (200a/c) (10 nM) and after 6 days the expression of E-CAD and GAPDH were studied by RT-qPCR. Non treated cells (NT); Mock-transfected cells (Mock). c Immunofluorescence staining of E-CAD (green) and texas-red phalloidin staining of F-ACTIN (red) in cells transfected as in (a) with scramble (siScr), pre-miR-200c and a ZEB1-specific siRNA pool (10 nM). Cells are counterstained with DAPI (blue) to visualize nuclei. Scale bars, $100 \mu \mathrm{m}$

transfected (cells treated with transfection reagent, no siRNA) and untransfected cells. Screening conditions were also optimized to ensure high transfection efficiency by using a siRNA pool targeting the essential gene, KIF11 (EG5) (data not shown).

To calculate E-CAD induction in the screen, a statistical method based on MAD calculation was used [21]. This method enabled a significant E-CAD induction detection of miR-200c and siZEB1 transfected cells. The MAD calculation method identified two groups of hit SMART pools. Group A contains 53 genes whose individual knockdown induced a statistically significant increase in E-CAD cellular fluorescence (threshold $\geq$ one $\mathrm{MAD}$ ) and morphological changes associated to a partial reversal of the mesenchymal phenotype and group B targeting 17 genes, which knockdown induced only morphological changes. Due to cell and siRNA transfection heterogeneity, we also considered these genes because they might be associated with modifications of adhesion properties and linked to metastatic process.

\section{Hit validation}

The fact that several target genes were already known to be involved, directly or indirectly, in MET conforted our strategy. These genes include in particular G9a [36], SMARCD3 [37], SMAD5 [38] and ZEB1, which is also the positive control (Fig. 3) [39]. We then focused on two genes: DOT1L (group B) and KAT5 (Tip60) (group A) (Fig. 4a and 5a). DOT1L is a histone H3 lysine 79 methyltransferase whose inhibition increases the yield of induced pluripotent stem cells (iPSCs) [40]. It was described very recently as an EMT modulator through a bioinformatic analysis of a large breast cancer genetic database [41]. KAT5 is an histone acetyltransferase (HAT) required to maintain characteristic features of ESCs [42]. It is linked for the first time here to the MET regulation. 

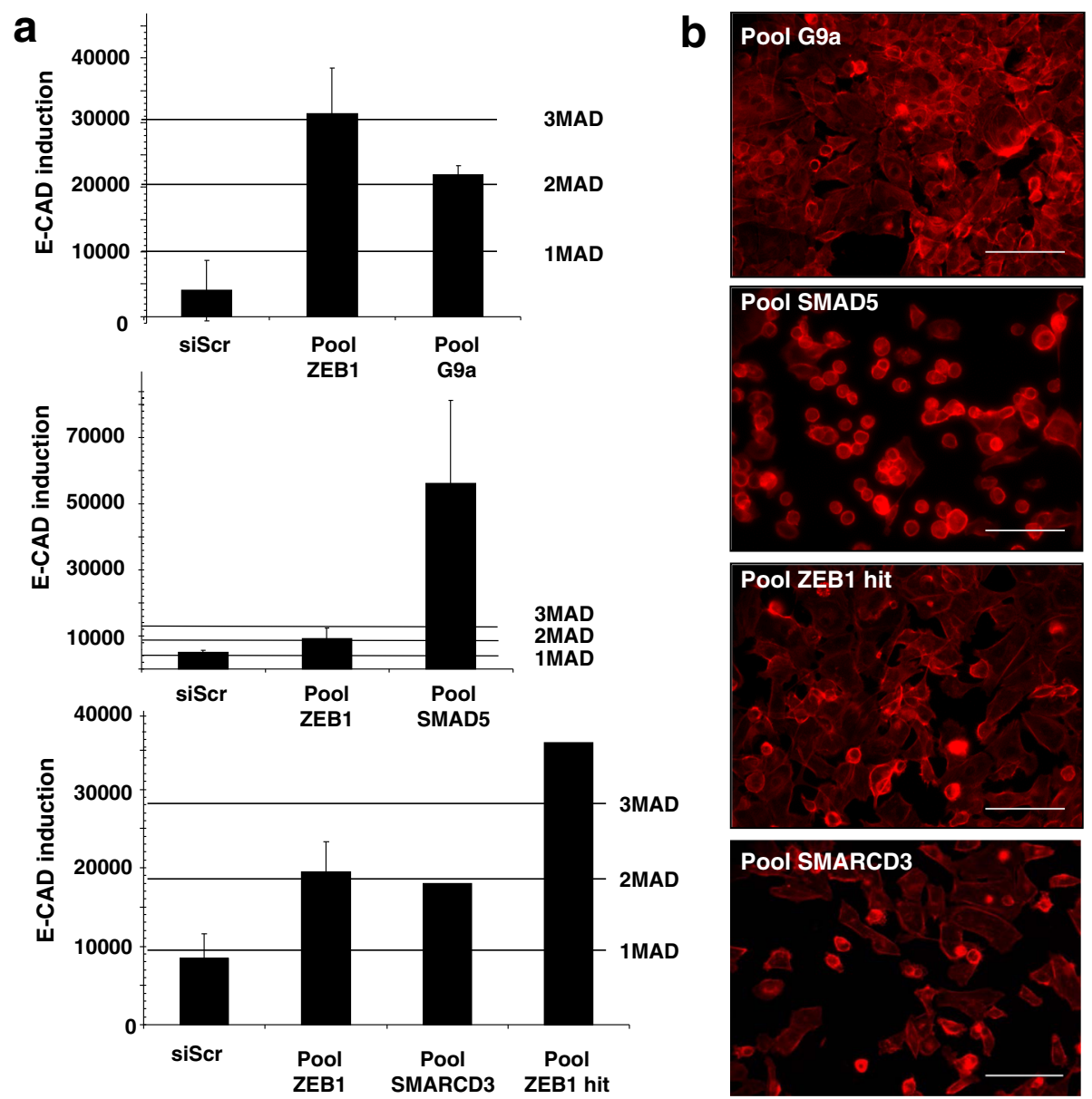

Fig. 3 High throughput functional screen to detect genes potentially involved in MET. a Four examples obtained in the initial screen. E-CAD expression was normalized to cell number then data were normalized to the median of SMARTpools in the same plate ( $n=80$ SMARTpools/plate). Threshold and hits selection were based on MAD calculation. b Morphological changes are revealed by F-ACTIN staining (red) as described before. Scale bars, $100 \mu \mathrm{m}$

To confirm the initial results and eliminate false positives due to off-target effects, we repeated the primary screen using deconvoluted single siRNAs targeting DOT1L and KAT5 (Figs. 4b and 5b). For each target, two out of four siRNAs tested present in the pools reproduced the observed primary screen phenotypes. Most remarkably, two out of four siRNAs targeting DOT1L were found to be significant E-CAD inducers when tested individually placing the DOT1L also in group A (Fig. 4b). The difference between the SMART pool and the single siRNA could be due to the siRNA potency. The effect of the DOT1L and KAT5 knockdown was further demonstrated by RT-qPCR and correlated to an increase in E-CAD mRNA and to a decrease in DOT1L or KAT5 mRNA levels. The implication of the two genes in MET regulation and stem/progenitor cell phenotypes was investigated by following the expression of mesenchymal and epithelial marker genes such as vimentin, ZEB1, E-cadherin, Tetraspanin 13 (TSPAN13),
Occludin $(O C L N)$ and the stem/progenitor cell surface markers CD24 and CD44. Among the seven markers studied, changes in $E-C A D$ and $C D 24$ expression were observed in response to DOT1L silencing and in $E-C A D$ and TSPAN13, a potent breast cancer suppressor gene [43], after KAT5 knockdown (Fig. 5c). The different marker expression profiles observed after DOT1L or KAT5 silencing may reflect partial MET [44].

A functional change associated with EMT is an increase in migration and/or invasion capacities [45]. As DOT1L or KAT5 silencing strongly decreases migration of MDA-MB-231, in vitro, after two successive transfections with no major effect on cell viability (Fig. 5d), we argued that DOT1L and KAT5 were involved in different steps of MDA-MB-231 differentiation and could be potential therapeutic targets to inhibit TNBC metastasis.

Finally, to confirm DOT1L as therapeutic target, we treated MDA-MB-231 cells with a potent and selective DOT1L inhibitor EPZ-5676 [46]. After a 7 days 


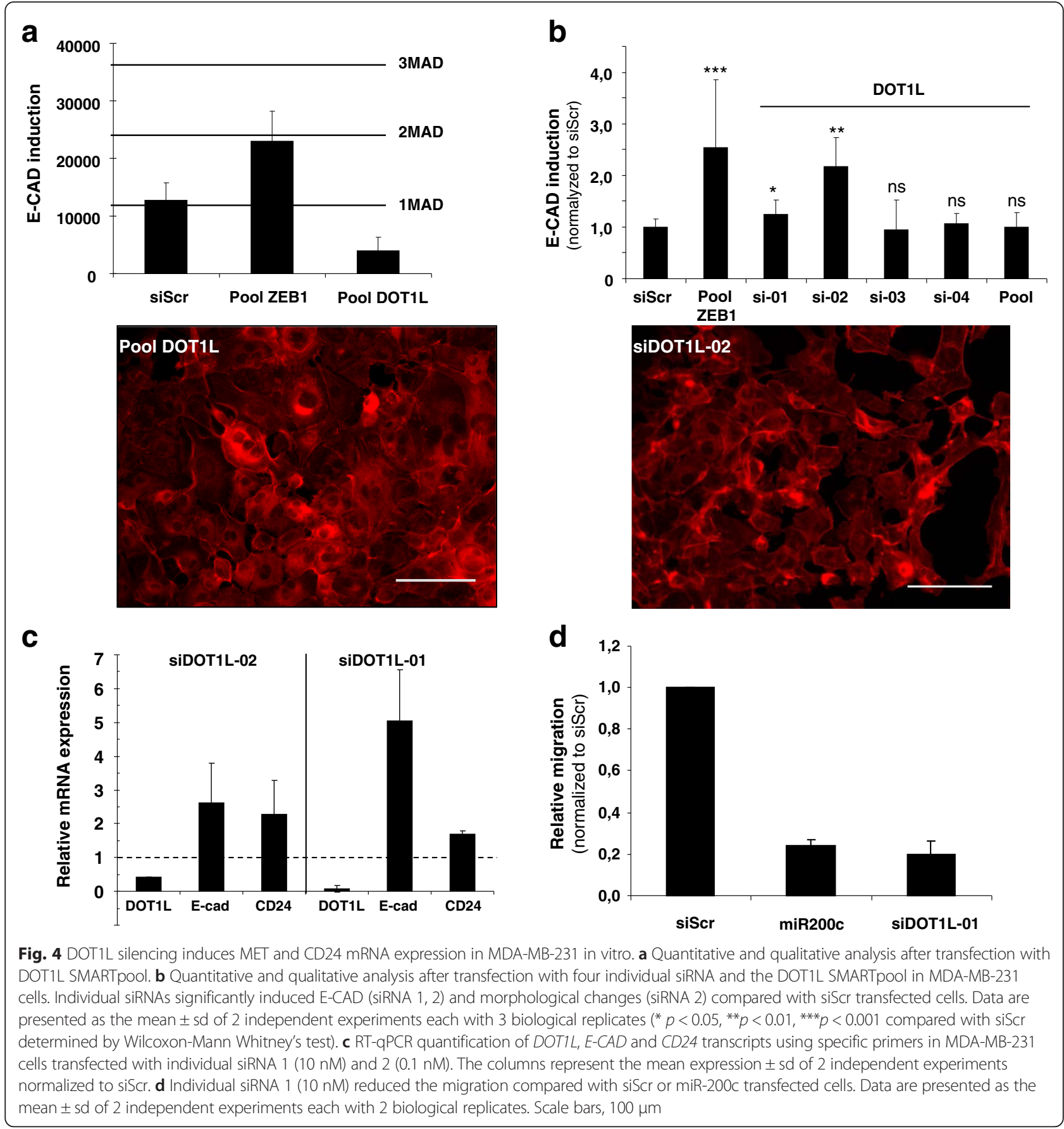

treatment, this drug showed a strong dose-dependent increase in E-CAD mRNA and a slight upregulation of CD24 mRNA (Fig. 6). These results were totally consistent with gene expression changes observed after DOT1L silencing and confirmed the role of DOT1L in MDAMB-231 CSC-like cells differentiation.

\section{Conclusions}

From this RNAi-based phenotypic screening, we have identified a set of 70 potentials hits, that may promote the conversion of the highly invasive mesenchymal-like cells MDA-MB-231 into a more differentiated and less aggressive phenotype. KAT5 and DOT1L gene downregulation induced E-CAD expression and epithelial morphological changes. The process was validated by the finding of hits such as ZEB1, G9a, SMAD5, SMARCD3, already reported in the literature to be implicated in the regulation of EMT/MET. Indeed, ZEB1 is a well known transcriptional repressor directly implicated in the control of EMT [34] that we used as positive control to 

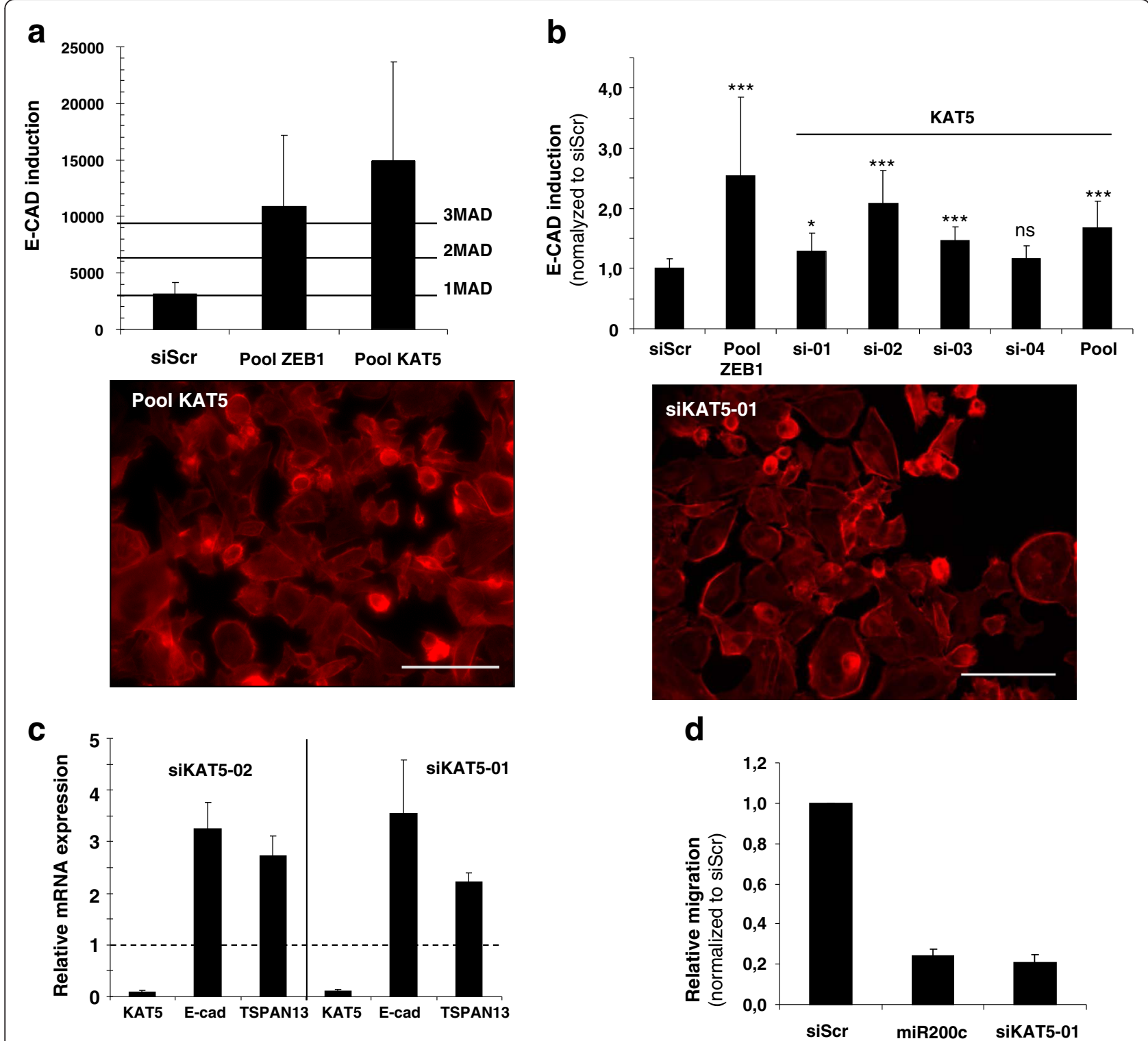

Fig. 5 KAT5 silencing induces MET and TSPAN13 mRNA expression in MDA-MB-231 in vitro. a Quantitative and qualitative analysis after transfection with KAT5 SMARTpool of E-CAD induction and cell morphology. $\mathbf{b}$ Quantitative and qualitative analysis after transfection with four individual siRNA and the KAT5 SMARTpool in MDA-MB-231 cells. Data are presented as the mean \pm sd of 2 independent experiments each with 3 biological replicates ( $p<0.05,{ }^{* *} p<0.01,{ }^{* * *} p<0.001$ compared with siScr determined by Wilcoxon-Mann Whitney's test). c RT-qPCR quantification of KAT5, E-CAD and TSPAN13 transcripts using specific primers in MDA-MB231 cells transfected with individual siRNA 1 (10nM) and 2 (1nM). The columns represent the mean expression \pm sd of 2 independent experiments normalized to siScr. $\mathbf{d}$ Individual siRNA 1 (10nM) reduced the migration compared with siScr or miR-200c transfected cells. Data are presented as the mean \pm sd of 2 independent experiments each with 2 biological replicates. Scale bars, $100 \mu \mathrm{m}$

design the screening assay. The knock-down of G9a, a histone methyltransferase, restored E-CAD expression, caused morphological changes and attenuated migratory and invasive capacity of MDA-MB-231 cell line in vitro and in vivo [36]. Furthermore, SMAD5 phosphorylation induced by an aberrant Aurora-A kinase activity, led to its nuclear activation and ultimately contributed to the development of EMT, stemness and tumor progression in human breast cancer cell line MCF-7 [38]. Finally, the silencing of SMARCD3/Baf60c, a SWI/SNF chromatin-remodeling factor, gives a strong MET by Wnt5a downregulation in EpCAM- SUM149 or SUM229 subpopulation [37].

Among the 70 gene candidate as putative MET regulators, DOT1L and KAT5 were found to both induce E$\mathrm{CAD}$ and to promote an epithelial morphological 

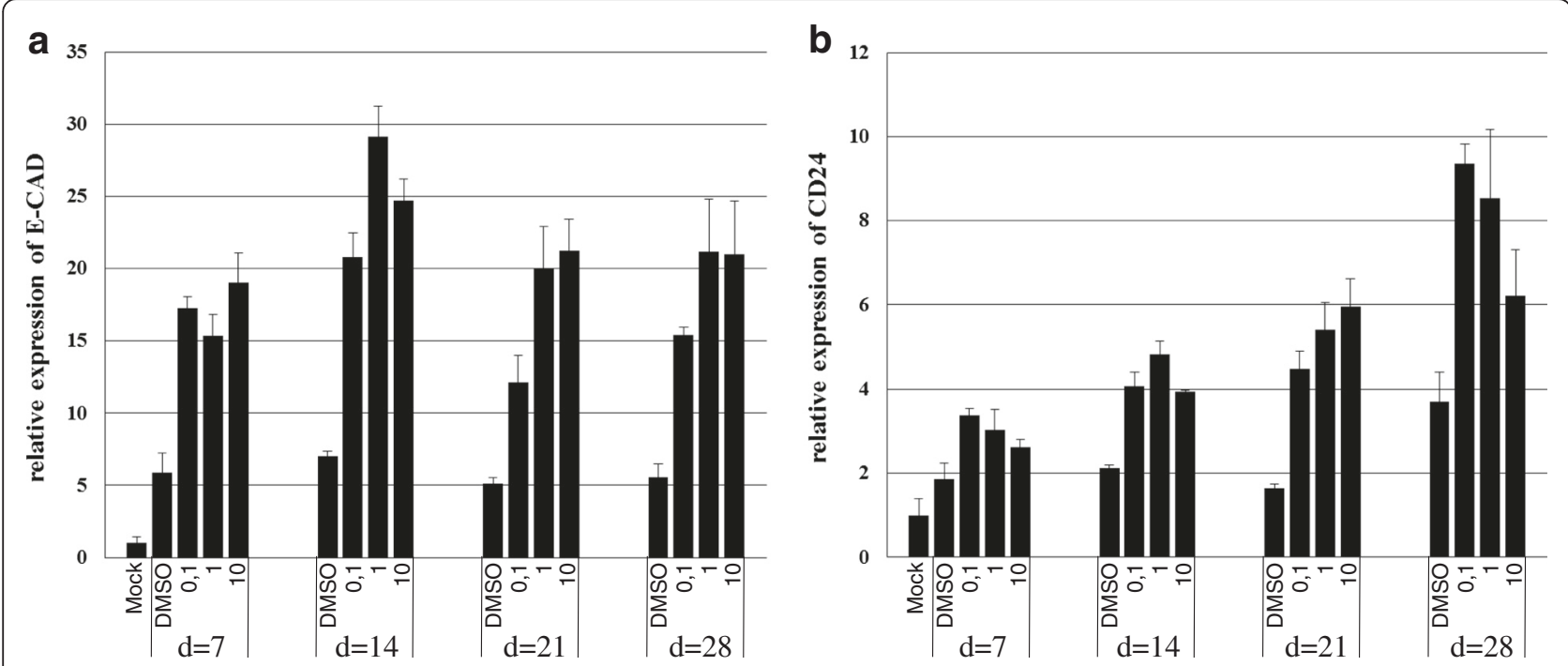

Fig. 6 Pharmacological inhibition of DOT1L induces E-cadherin and CD24 expression in MDA-MB-231 in vitro. Cells were treated with dose effects of EPZ-5676 $(0.1 \mu \mathrm{M}, 1 \mu \mathrm{M}, 10 \mu \mathrm{M})$ or $0.1 \%$ DMSO for the indicated days (noted d), followed by mRNA extraction and RT-qPCR with specific primers. a E-CAD expression relative to non treated cells. $\mathbf{b}$ CD24 expression relative to non treated cells. Data in $\mathbf{a}$ and $\mathbf{b}$ are the mean of RT-qPCR replicates from a representative experiment, and error bars indicate SEM. The experiments were run 2 times

phenotype in MDA-MB-231. DOT1L was previously identified as a modulator of pluripotent stem cells (iPSCs) reprogramming [40] and shown to methylated the H3K79 mark which is critical in Mixed Lineage leukemia (MLL) by enhancing expression of leukemogenic genes like HOXA9 and MEIS1 [47]. In vivo, administration of a DOT1L selective inhibitor increased the lifespan of mice grafted with a preclinical model of MLL [46]. In colon cancer, DOT1L increases cancer stemness and tumorigenic potential by inducing the core stem cell genes NANOG, SOX2 and Pou5F1 [48]. In this study, DOT1L silencing and chemical inhibition by EPZ5676 induced E-CAD and $\mathrm{CD} 24$ expression and reduced the migration properties of MDA-MB-231 cells. These results support the idea that DOT1L is involved in EMT and in the maintenance of $\mathrm{CD}_{4} 4^{+} / \mathrm{CD} 24^{-}$cancer stem cells present in MDA-MB-231 cell line. These results are in agreement with those published by Zhang et al. in 2014, showing that DOT1L was a potential drug target for breast cancer and metastatic disease [41].

Finally, this siRNA screening led to the identification of KAT5, a target never described in MET regulation up today. KAT5 is a HAT with regulatory functions in signalling, transcriptional activation, DNA repair, apoptosis and cell cycle progression [49]. In embryonic stem cells (ESCs), one of the most important functions of KAT5 is to repress developmental genes [42]. In basal-like breast cancer, the TWIST protein, a well known EMT inducer [50], is specifically diacetylated by KAT5 to interact with BRD4 and activate WNT5A. As a result of this interaction, it induces invasion and increases (CSC)-like properties and tumorigenicity. Lastly, in radioresistant subpopulations of breast cancer cells induced by irradiation, ATM, a protein activated by KAT5 acetylation, is hyperactivated and mediates stabilization of ZEB1, another well known EMT inducer, in breast cancer and other types of solid tumours [51, 52]. Altogether, combined with the fact that KAT5 silencing induces E-CAD and TSPAN13 expression, it strongly suggests that a KAT5 inhibitor can induce TNBC differentiation (basal-like subtype) and, in combination with classical chemotherapeutic agents, reduces the number of metastases [53]. Another study shows a metastatic suppression function of KAT5 in a prostate cancer model highlighting the fact that EMT regulation is strongly tissue dependant [54]. Moreover, as a result of the direct relationship between KAT5 and ATM kinase, our findings may highlight the critical role of the DNA damage response (DDR) in tumorigenesis and metastasis in the basal subtype of breast cancer $[55,56]$.

In conclusion, the screening method we developed enables the identification of validated and putative targets involved in the mesenchyme phenotype maintenance of triple negative breast cancer cells. These targets need to be further investigated to demonstrate their antitumoral effect in animal models and patients. 


\section{Additional file}

Additional file 1: Raw Data. An excel file containing 10 spreadsheets. Each spreadsheet has the name of the figure in which the data were used. (XLS $788 \mathrm{~kb}$ )

\section{Acknowledgements}

The RNAi screening experiments were carried out on the "Plateforme Intégrée de Criblage de Toulouse" (PICT, IBISA) facilities.

\section{Funding}

This work was funded by the Institut de Recherche Pierre Fabre and the Centre national de la Recherche Scientifique.

\section{Availability of data and materials}

All data generated or analysed during this study are included in this published article as a Additional file 1 .

\section{Authors' contributions}

JMG conceived the study, carried out the experiments and wrote the manuscript, LF participated in the RT-qPCR experiments and drafted the manuscript. CSC and VM participated in the whole screening experiments. FAl designed the cell morphology analysis experiments. PBA supported the study, and participated in its coordination. FAu participated in the study design and coordination and wrote the manuscript. All authors read and approved the final manuscript.

\section{Competing interests}

The authors declare that they have no competing interests.

\section{Consent for publication}

Not applicable.

\section{Ethics approval and consent to participate}

Not applicable.

Received: 1 July 2015 Accepted: 5 August 2016

Published online: 31 August 2016

\section{References}

1. Mani SA, Guo W, Liao MJ, Eaton EN, Ayyanan A, Zhou AY, Brooks M, Reinhard F, Zhang CC, Shipitsin M, et al. The epithelial-mesenchymal transition generates cells with properties of stem cells. Cell. 2008;133(4):704-15.

2. Kalluri R, Weinberg RA. The basics of epithelial-mesenchymal transition. I Clin Invest. 2009:119(6):1420-8.

3. Thiery JP, Acloque H, Huang RY, Nieto MA. Epithelial-mesenchymal transitions in development and disease. Cell. 2009;139(5):871-90.

4. Al-Hajj M, Wicha MS, Benito-Hernandez A, Morrison SJ, Clarke MF. Prospective identification of tumorigenic breast cancer cells. Proc Natl Acad Sci U S A. 2003;100(7):3983-8.

5. Wicha MS, Liu S, Dontu G. Cancer stem cells: an old idea-a paradigm shift Cancer Res. 2006;66(4):1883-90. discussion 1895-1886.

6. Dean M, Fojo T, Bates S. Tumour stem cells and drug resistance. Nat Rev Cancer. 2005:5(4):275-84

7. Gunasinghe NP, Wells A, Thompson EW, Hugo HJ. Mesenchymal-epithelial transition (MET) as a mechanism for metastatic colonisation in breast cancer. Cancer Metastasis Rev. 2012;31(3-4):469-78.

8. Bonnomet A, Syne L, Brysse A, Feyereisen E, Thompson EW, Noel A, Foidart JM, Birembaut P, Polette M, Gilles C. A dynamic in vivo model of epithelial-tomesenchymal transitions in circulating tumor cells and metastases of breast cancer. Oncogene. 2012;31(33):3741-53.

9. Thiery JP, Lim CT. Tumor dissemination: an EMT affair. Cancer Cell. 2013;23(3):272-3.

10. Yu M, Bardia A, Wittner BS, Stott SL, Smas ME, Ting DT, Isakoff SJ, Ciciliano JC, Wells MN, Shah AM, et al. Circulating breast tumor cells exhibit dynamic changes in epithelial and mesenchymal composition. Science. 2013; 339(6119):580-4.

11. Nickel A, Stadler SC. Role of epigenetic mechanisms in epithelial-to-mesenchymal transition of breast cancer cells. Transl Res. 2015;165(1):126-42.

12. Kouzarides T. Chromatin modifications and their function. Cell. 2007;128(4):693-705.
13. Dixon JR, Jung I, Selvaraj S, Shen Y, Antosiewicz-Bourget JE, Lee AY, Ye Z, Kim A, Rajagopal N, Xie W, et al. Chromatin architecture reorganization during stem cell differentiation. Nature. 2015;518(7539):331-6.

14. Polak P, Karlic R, Koren A, Thurman R, Sandstrom R, Lawrence MS, Reynolds A, Rynes E, Vlahovicek K, Stamatoyannopoulos JA, et al. Cell-of-origin chromatin organization shapes the mutational landscape of cancer. Nature. 2015;518(7539):360-4.

15. Cerone MA, Burgess DJ, Naceur-Lombardelli C, Lord CJ, Ashworth A. Highthroughput RNAi screening reveals novel regulators of telomerase. Cancer Res. 2011;71(9):3328-40.

16. Stec E, Locco L, Szymanski S, Bartz SR, Toniatti C, Needham RH, Palmieri A, Carleton M, Cleary MA, Jackson AL, et al. A multiplexed siRNA screening strategy to identify genes in the PARP pathway. J Biomol Screen. 2012;17(10):1316-28.

17. Iorns E, Lord CJ, Turner N, Ashworth A. Utilizing RNA interference to enhance cancer drug discovery. Nat Rev Drug Discov. 2007;6(7):556-68.

18. lorns E, Turner NC, Elliott R, Syed N, Garrone O, Gasco M, Tutt AN, Crook T, Lord CJ, Ashworth A. Identification of CDK10 as an important determinant of resistance to endocrine therapy for breast cancer. Cancer Cell. 2008;13(2):91-104.

19. Pu S, Turinsky AL, Vlasblom J, On T, Xiong X, Emili A, Zhang Z, Greenblatt J, Parkinson J, Wodak SJ. Expanding the landscape of chromatin modification (CM)-related functional domains and genes in human. PLoS One. 2010;5(11):e14122.

20. Park SM, Gaur AB, Lengyel E, Peter ME. The miR-200 family determines the epithelial phenotype of cancer cells by targeting the E-cadherin repressors ZEB1 and ZEB2. Genes Dev. 2008;22(7):894-907.

21. Chung N, Zhang XD, Kreamer A, Locco L, Kuan PF, Bartz S, Linsley PS, Ferrer M, Strulovici B. Median absolute deviation to improve hit selection for genome-scale RNAi screens. J Biomol Screen. 2008;13(2):149-58.

22. Moreno-Bueno G, Peinado H, Molina P, Olmeda D, Cubillo E, Santos V, Palacios J, Portillo F, Cano A. The morphological and molecular features of the epithelial-to-mesenchymal transition. Nat Protoc. 2009;4:1591-1613.

23. Boutros $M$, Ahringer J. The art and design of genetic screens: RNA interference. Nat Rev Genet. 2008;9:554-566.

24. Lall S. Primers on chromatin. Nat Struct Mol Biol. 2007;14(11):1110-5.

25. Taverna SD, Li H, Ruthenburg AJ, Allis CD, Patel DJ. How chromatin-binding modules interpret histone modifications: lessons from professional pocket pickers. Nat Struct Mol Biol. 2007;14(11):1025-40.

26. Shi J, Vakoc CR. The mechanisms behind the therapeutic activity of BET bromodomain inhibition. Mol Cell. 2014;54(5):728-36.

27. Finn RD, Mistry J, Schuster-Bockler B, Griffiths-Jones S, Hollich V, Lassmann T, Moxon S, Marshall M, Khanna A, Durbin R, et al. Pfam: clans, web tools and services. Nucleic Acids Res. 2006;34(Database issue):D247-251.

28. Lombaerts M, van Wezel T, Philippo K, Dierssen JW, Zimmerman RM, Oosting J, van Eijk R, Eilers PH, van de Water B, Cornelisse CJ, et al. E-cadherin transcriptional downregulation by promoter methylation but not mutation is related to epithelial-to-mesenchymal transition in breast cancer cell lines. Br J Cancer. 2006;94(5):661-71.

29. Law ME, Corsino PE, Jahn SC, Davis BJ, Chen S, Patel B, Pham K, Lu J, Sheppard B, Norgaard P, et al. Glucocorticoids and histone deacetylase inhibitors cooperate to block the invasiveness of basal-like breast cancer cells through novel mechanisms. Oncogene. 2013;32(10):1316-29.

30. Tate CR, Rhodes LV, Segar HC, Driver JL, Pounder FN, Burow ME, Collins-Burow BM. Targeting triple-negative breast cancer cells with the histone deacetylase inhibitor panobinostat. Breast Cancer Res. 2012;14(3):R79.

31. Sheridan C, Kishimoto H, Fuchs RK, Mehrotra S, Bhat-Nakshatri P, Turner CH, Goulet Jr R, Badve S, Nakshatri H. CD44+/CD24- breast cancer cells exhibit enhanced invasive properties: an early step necessary for metastasis. Breast Cancer Res. 2006;8(5):R59.

32. Lu M, Jolly MK, Onuchic J, Ben-Jacob E. Toward decoding the principles of cancer metastasis circuits. Cancer Res. 2014;74(17):4574-87.

33. Burk U, Schubert J, Wellner U, Schmalhofer O, Vincan E, Spaderna S, Brabletz T. A reciprocal repression between $\mathrm{ZEB} 1$ and members of the miR-200 family promotes EMT and invasion in cancer cells. EMBO Rep. 2008;9(6):582-9.

34. Eger A, Aigner K, Sonderegger S, Dampier B, Oehler S, Schreiber M, Berx G, Cano A, Beug H, Foisner R. DeltaEF1 is a transcriptional repressor of E-cadherin and regulates epithelial plasticity in breast cancer cells. Oncogene. 2005;24(14):2375-85.

35. Gregory PA, Bert AG, Paterson EL, Barry SC, Tsykin A, Farshid G, Vadas MA Khew-Goodall Y, Goodall GJ. The miR-200 family and miR-205 regulate epithelial to mesenchymal transition by targeting ZEB1 and SIP1. Nat Cell Biol. 2008;10(5):593-601. 
36. Dong C, Wu Y, Yao J, Wang Y, Yu Y, Rychahou PG, Evers BM, Zhou BP. G9a interacts with Snail and is critical for Snail-mediated E-cadherin repression in human breast cancer. J Clin Invest. 2012;122(4):1469-86.

37. Jordan NV, Prat A, Abell AN, Zawistowski JS, Sciaky N, Karginova OA, Zhou B, Golitz BT, Perou CM, Johnson GL. SWI/SNF chromatin-remodeling factor Smarcd3/Baf60c controls epithelial-mesenchymal transition by inducing Wnt5a signaling. Mol Cell Biol. 2013;33(15):3011-25.

38. D'Assoro AB, Liu T, Quatraro C, Amato A, Opyrchal M, Leontovich A, Ikeda Y, Ohmine S, Lingle W, Suman V, et al. The mitotic kinase Aurora-a promotes distant metastases by inducing epithelial-to-mesenchymal transition in ERalpha(+) breast cancer cells. Oncogene. 2014;33(5):599-610.

39. Brabletz S, Brabletz T. The ZEB/miR-200 feedback loop-a motor of cellular plasticity in development and cancer? EMBO Rep. 2010;11(9):670-7.

40. Onder TT, Kara N, Cherry A, Sinha AU, Zhu N, Bernt KM, Cahan P, Marcarci BO, Unternaehrer J, Gupta PB, et al. Chromatin-modifying enzymes as modulators of reprogramming. Nature. 2012;483(7391):598-602.

41. Zhang L, Deng L, Chen F, Yao Y, Wu B, Wei L, Mo Q, Song Y. Inhibition of histone H3K79 methylation selectively inhibits proliferation, self-renewal and metastatic potential of breast cancer. Oncotarget. 2014;5(21):10665-77.

42. Fazzio TG, Huff JT, Panning B. Chromatin regulation Tip(60)s the balance in embryonic stem cell self-renewal. Cell Cycle. 2008;7(21):3302-6.

43. Huang H, Sossey-Alaoui K, Beachy SH, Geradts J. The tetraspanin superfamily member NET-6 is a new tumor suppressor gene. J Cancer Res Clin Oncol. 2007;133(10):761-9.

44. Tam WL, Weinberg RA. The epigenetics of epithelial-mesenchymal plasticity in cancer. Nat Med. 2013:19(11):1438-49.

45. Lim J, Thiery JP. Epithelial-mesenchymal transitions: insights from development. Development. 2012;139(19):3471-86.

46. Daigle SR, Olhava EJ, Therkelsen CA, Basavapathruni A, Jin L, Boriack-Sjodin PA, Allain CJ, Klaus CR, Raimondi A, Scott MP, et al. Potent inhibition of DOT1L as treatment of MLL-fusion leukemia. Blood. 2013;122(6):1017-25.

47. Nguyen AT, Taranova O, He J, Zhang Y. DOT1L, the H3K79 methyltransferase, is required for MLL-AF9-mediated leukemogenesis. Blood. 2011;117(25):6912-22.

48. Kryczek I, Lin Y, Nagarsheth N, Peng D, Zhao L, Zhao E, Vatan L, Szeliga W, Dou Y, Owens S, et al. IL-22(+)CD4(+) T cells promote colorectal cancer stemness via STAT3 transcription factor activation and induction of the methyltransferase DOT1L. Immunity. 2014;40(5):772-84.

49. Squatrito M, Gorrini C, Amati B. Tip60 in DNA damage response and growth control: many tricks in one HAT. Trends Cell Biol. 2006;16(9):433-42.

50. Puisieux A, Brabletz T, Caramel J. Oncogenic roles of EMT-inducing transcription factors. Nat Cell Biol. 2014;16(6):488-94.

51. Zhang P, Wei Y, Wang L, Debeb BG, Yuan Y, Zhang J, Yuan J, Wang M, Chen D, Sun Y, et al. ATM-mediated stabilization of ZEB1 promotes DNA damage response and radioresistance through CHK1. Nat Cell Biol. 2014;16(9):864-75.

52. Schmalhofer O, Brabletz S, Brabletz T. E-cadherin, beta-catenin, and ZEB1 in malignant progression of cancer. Cancer Metastasis Rev. 2009;28(1-2):151-66.

53. Tsai JH, Yang J. Epithelial-mesenchymal plasticity in carcinoma metastasis. Genes Dev. 2013:27(20):2192-206.

54. Kim JH, Kim B, Cai L, Choi HJ, Ohgi KA, Tran C, Chen C, Chung CH, Huber O, Rose DW, et al. Transcriptional regulation of a metastasis suppressor gene by Tip60 and beta-catenin complexes. Nature. 2005:434(7035):921-6.

55. Lehmann BD, Bauer JA, Chen X, Sanders ME, Chakravarthy AB, Shyr Y, Pietenpol JA. Identification of human triple-negative breast cancer subtypes and preclinical models for selection of targeted therapies. J Clin Invest. 2011;121(7):2750-67.

56. Sun M, Guo X, Qian X, Wang H, Yang C, Brinkman KL, Serrano-Gonzalez M, Jope RS, Zhou B, Engler DA, et al. Activation of the ATM-Snail pathway promotes breast cancer metastasis. J Mol Cell Biol. 2012;4(5):304-15.

\section{Submit your next manuscript to BioMed Central and we will help you at every step:}

- We accept pre-submission inquiries

- Our selector tool helps you to find the most relevant journal

- We provide round the clock customer support

- Convenient online submission

- Thorough peer review

- Inclusion in PubMed and all major indexing services

- Maximum visibility for your research

Submit your manuscript at www.biomedcentral.com/submit
() BioMed Central 